\title{
Gesundheitsversorgung - die Schweiz im internationalen Vergleich
}

Gerhard Kocher

Korrespondenz:

Dr. rer. pol. Gerhard Kocher

Haldenweg $10 \mathrm{~A}$

CH-3074 Muri

gerhard.kocher[at]muri-be.ch
Seit 2004 präsentiert die SÄZ die jährliche Daten-CD mit dem Titel «OECD Health Data». Herausgeberin ist die Organisation für wirtschaftliche Zusammenarbeit und Entwicklung in Paris, der heute 34 Staaten angehören. Von ihren rund 2500 Mitarbeiterinnen und Mitarbeitern sind etwa 1600 Experten. Die Jahres-CD erscheint leider nicht mehr, sondern nur noch die jährliche Broschüre «Health at a glance» [1]. Sie ist eine kommentierte Auswahl von 200 Grafiken auf der Basis der OECD-Datenbank. Die Broschüre ist zu empfehlen, enthält aber viel weniger Daten als die CD, die 85 Euro kostete. Der Zugriff auf die Gesundheitsdatenbank der OECD [2] kostet jährlich 308 Euro. Er ist viel komplizierter als auf die gutprogrammierten früheren CDs. Etwa 55 Excel-Tabellen sind als Key Indicators immerhin kostenlos abrufbar (www.oecd.org $\rightarrow$ Topics $\rightarrow$ Health). Insgesamt ist die Abschaffung der CD ein unverständlicher und kundenunfreundlicher Fehlentscheid, der ausgerechnet die weltweit wohl wichtigste Datenquelle zum Vergleich verschiedener Gesundheitssysteme betrifft.

Die hier kommentierten Daten betreffen 2009 und stammen aus «Health at a glance 2011», den «Key Indicators», der OECD-Datenbank und aus eigenen Berechnungen.

\section{Wie gewohnt:}

\section{exorbitante Gesundheitsausgaben der USA}

Die Tabelle 1 zeigt im internationalen Vergleich einmal mehr, wie schwer das US-Gesundheitssystem die Bürger, den Staat und die Wirtschaft belastet bei einem keineswegs adäquaten Leistungs-KostenVerhältnis. Die Pro-Kopf-Ausgaben (Spalten 2 und 3) der USA sind 184\% höher als der OECD-Durchschnitt und 55\% höher als die Ausgaben der Schweiz im dritten Rang. In den neunziger Jahren war die Schweiz immer im zweiten Rang (nach den USA). Seit 2000 erreichte unser Land nur einmal den zweiten Rang, fünfmal den dritten und viermal den vierten Rang. Zwischen die USA und die Schweiz schob sich jeweils Norwegen oder Luxemburg. Von 2000 bis 2009 nahmen die Ausgaben per capita (kaufkraftbereinigt) im OECD-Schnitt um $81 \%$ zu, in den USA um 69 und in der Schweiz um 60\%. Laut BAG kostete 2009 unser Gesundheitswesen 7827 Franken pro Person [3].

Bei internationalen Vergleichen der Gesundheitsausgaben zieht man seit jeher nicht nur die ProKopf-Ausgaben heran, sondern auch den BIP-Anteil (Spalte 4): Der Vergleich der Gesundheitsausgaben mit dem jeweiligen Bruttoinlandprodukt BIP, dem
Gesamtwert aller Waren und Dienstleistungen, die im betreffenden Jahr innerhalb der Landesgrenzen hergestellt wurden und dem Endverbrauch dienen. So gemessen ist die Diskrepanz geringer. Die US-Ausgaben sind aber immer noch $98 \%$ höher als der OECD-Durchschnitt und 53\% höher als die Schweizer Gesundheitsausgaben. Bei den Zahlen in dieser Spalte ist zu beachten, dass sie als Verhältniszahlen stark von der Wirtschaftsentwicklung im betreffenden Land und Jahr abhängen. Eine Zunahme beispielsweise kann durch eine schwächere Konjunktur verursacht sein und nicht (allein) durch höhere Gesundheitsausgaben. Von 2000 bis 2009 stieg der BIP-Anteil im Durchschnitt von 40 Ländern von 7,9 auf $9,6 \%$, also um $21,5 \%$ (USA $27 \%$, Schweiz $11,8 \%$ ).

Die Ländervergleiche der Entwicklung der Gesundheitskosten sowohl in Dollar pro Kopf als auch in Prozent vom BIP zeigen deutlich, warum die Gesundheitskosten in allen Ländern ein politisches Hauptproblem sind. Die zahllosen Sparmassnahmen sind nicht nur höchst umstritten, sondern wirken nur begrenzt. Die im November 2011 veröffentlichte KOF-Prognose [4] nimmt an, dass die Schweizer Gesundheitsausgaben von 2009 auf 2010 um 4,4\% gestiegen sind, von 2010 auf 2011 um 2,7\% und von 2011 auf 2012 um 3,2\%. Danach kostet unser Gesundheitssystem jedes Jahr 1,7 bis 2 Milliarden Franken mehr als im Vorjahr. 2013 soll die Zunahme sogar 2,3 Milliarden betragen. Für 2012 schätzt die KOF die Ausgaben auf 66,6 Milliarden Franken, für 2013 auf 68,9 Milliarden.

Die Kostensteigerung bleibt also ein reales Problem und lässt sich nicht mit Lobreden über die blühende «Gesundheitswirtschaft» verharmlosen. Und noch weniger mit der interessengesteuerten, oft penetranten Heiligsprechung der Gesundheitsausgaben.

\section{Sonderfall Schweiz: sehr hohe Direktzahlungen der Patienten}

In internationalen Vergleichen fallen immer die hohen Out-of-pocket-Zahlungen in der Schweiz auf (Tab. 2). Sie betreffen vor allem Leistungen für Pflegeheime, Institutionen für Behinderte, praktisch die ganze Zahnmedizin, rezeptfreie Medikamente sowie Franchisen und Selbstbehalte. Gesamthaft zahlen die Schweizer Haushalte neben den Versicherungsprämien 30\% der gesamten Gesundheitsausgaben direkt aus eigener Tasche. Die beiden anderen Finanzierungsquellen sind die Sozialversicherungen mit $40,8 \%$ und der Staat mit 19,4\% [5]. 
Tabelle 1

Gesundheitsausgaben pro Kopf und in Prozent vom BIP. Ausgewählte Länder 2009, teilweise 2008. OECD und eigene Berechnungen.

\begin{tabular}{|c|c|c|c|c|}
\hline & $\begin{array}{l}\text { US-\$, } \\
\text { kaufkraft- } \\
\text { bereinigt }\end{array}$ & $\begin{array}{l}\text { Index: } \\
\text { Durchschnitt } \\
\text { aller Länder } \\
=\text { Index } 100\end{array}$ & $\begin{array}{l}\text { Prozent vom } \\
\text { Bruttoinland- } \\
\text { produkt }\end{array}$ & $\begin{array}{l}\text { Index: } \\
\text { Durchschnitt } \\
\text { aller Länder } \\
=\text { Index } 100\end{array}$ \\
\hline USA & 7960 & 284 & 17,4 & 198 \\
\hline Norwegen & 5352 & 191 & 9,6 & 109 \\
\hline Schweiz & 5144 & 183 & 11,4 & 130 \\
\hline Niederlande & 4914 & 175 & 12 & 136 \\
\hline Luxemburg & 4808 & 171 & 11,4 & 130 \\
\hline Kanada & 4363 & 156 & 11,5 & 131 \\
\hline Dänemark & 4348 & 155 & 11 & 125 \\
\hline Österreich & 4289 & 153 & 11,6 & 132 \\
\hline Deutschland & 4218 & 150 & 11,8 & 134 \\
\hline Frankreich & 3978 & 142 & 9,5 & 108 \\
\hline Irland & 3781 & 135 & 7,8 & 89 \\
\hline Schweden & 3722 & 133 & 10 & 114 \\
\hline Island & 3538 & 126 & 9,7 & 110 \\
\hline Grossbritannien & 3487 & 124 & 9,8 & 111 \\
\hline Australien & 3445 & 123 & 8,7 & 99 \\
\hline Finnland & 3226 & 115 & 9,2 & 105 \\
\hline Italien & 3137 & 112 & 9,5 & 108 \\
\hline Spanien & 3067 & 109 & 9,5 & 108 \\
\hline Neuseeland & 2983 & 106 & 10,3 & 117 \\
\hline Slowenien & 2579 & 92 & 7,9 & 90 \\
\hline Portugal & 2508 & 89 & 8,2 & 93 \\
\hline Israel & 2165 & 77 & 9,1 & 103 \\
\hline Tschechien & 2108 & 75 & 6,9 & 78 \\
\hline Slowakei & 2084 & 74 & 7,4 & 84 \\
\hline Südkorea & 1879 & 67 & 7,4 & 84 \\
\hline Ungarn & 1511 & 54 & 7 & 80 \\
\hline Polen & 1394 & 50 & 7 & 80 \\
\hline Estland & 1393 & 50 & 8,4 & 95 \\
\hline Chile & 1186 & 42 & 5,4 & 61 \\
\hline Russland & 1036 & 37 & 9 & 102 \\
\hline Brasilien & 943 & 34 & 6,4 & 73 \\
\hline Mexiko & 918 & 33 & 6,1 & 69 \\
\hline Türkei & 902 & 32 & 8,5 & 97 \\
\hline Südafrika & 862 & 31 & 4,6 & 52 \\
\hline China & 308 & 11 & 4,2 & 48 \\
\hline Indien & 132 & 5 & 2,4 & 27 \\
\hline Indonesien & 99 & 4 & 8,4 & 95 \\
\hline Durchschnitt & 2805 & 100 & 8,8 & 100 \\
\hline
\end{tabular}

Die Schweiz ist hier mit grossem Vorsprung im ersten Rang: Die 1568 US-Dollar pro Person und Jahr sind $61 \%$ höher als die Direktzahlungen in den USA und 177\% höher als der OECD-Durchschnitt. Die Deutschen zahlen nur 552 US-Dollar direkt, die Franzosen sogar nur 291 US-Dollar. Wenn man die unterschiedliche Kaufkraft der Länder nicht berücksichtigt, sondern die Beträge zum Wechselkurs ver- gleicht, ist die Diskrepanz noch grösser. Die Selbstzahlungen der Schweizer Privathaushalte entsprechen 3,5\% des Bruttoinlandprodukts. Das ist fast der doppelte OECD-Durchschnitt. Kein anderes Land in Europa ausser der Slowakei zahlt mehr als 1,9\% [6].

\section{Zahl der Ärzte und Pflegefachpersonen}

Im Durchschnitt der OECD-Länder entfielen auf 1000 Einwohner 2,8 Ärzte und 7,7 Pflegefachleute (Tab. 3). Die Ärztedichte (praktizierende Ärzte) ist in der Schweiz 36\% höher $(3,8)$, die Pflegendendichte (praktizierende Pflegende) ist sogar doppelt so hoch $(15,2)$. Schon im Jahr 2000 hatten wir 84\% mehr Pflegende pro 1000 Einwohner als der OECD-Durchschnitt. Das Zahlenverhältnis Pflegende/Ärzte ist bei uns nach Indonesien, USA, Dänemark und Japan $(4,3)$ das höchste $(4$, wie Luxemburg und Neuseeland).

Tabelle 2

Selbstzahlungen der Privathaushalte pro Kopf, 2009. Australien, Japan, Portugal: 2008.

\begin{tabular}{|c|c|c|}
\hline & $\begin{array}{l}\text { US-\$, } \\
\text { kaufkraftbereinigt }\end{array}$ & In \% vom BIP \\
\hline Schweiz & 1568 & 3,5 \\
\hline USA & 976 & 2,1 \\
\hline Norwegen & 808 & 1,5 \\
\hline Belgien & 787 & 1,7 \\
\hline Portugal & 681 & \\
\hline Kanada & 636 & 1,7 \\
\hline Australien & 627 & \\
\hline Israel & 623 & 2,3 \\
\hline Schweden & 620 & 1,7 \\
\hline Italien & 617 & 1,9 \\
\hline Spanien & 616 & 1,9 \\
\hline Finnland & 614 & 1,7 \\
\hline Südkorea & 609 & 2,2 \\
\hline Island & 588 & 1,2 \\
\hline Dänemark & 573 & \\
\hline Luxemburg & 559 & 0.9 \\
\hline Deutschland & 552 & 1,5 \\
\hline Slowakei & 533 & 2,3 \\
\hline Irland & 464 & 1,2 \\
\hline Japan & 454 & \\
\hline Mexiko & 439 & 3,1 \\
\hline Chile & 403 & 2,9 \\
\hline Neuseeland & 399 & 1,4 \\
\hline Grossbritannien & 365 & 1 \\
\hline Ungarn & 359 & 1,8 \\
\hline Slowenien & 334 & 1,2 \\
\hline Polen & 310 & 1,6 \\
\hline Tschechien & 304 & 1,7 \\
\hline Frankreich & 291 & 0,9 \\
\hline Estland & 282 & 1,4 \\
\hline Durchschnitt & 566 & 1,8 \\
\hline
\end{tabular}


Tabelle 3

Praktizierende Ärzte und praktizierendes Pflegepersonal pro 1000 Einwohner, 2009.

\begin{tabular}{|c|c|c|c|}
\hline & Ärzte & Pflegepersonen & $\begin{array}{l}\text { Verhältnis } \\
\text { Pflegepersonen/ Ärzte }\end{array}$ \\
\hline Österreich & 4,7 & 7,6 & 1,6 \\
\hline Russland & 4,3 & 8,1 & 1,9 \\
\hline Norwegen & 4 & 14,2 & 3,6 \\
\hline Schweiz & 3,8 & 15,2 & 4,0 \\
\hline Tschechien & 3,8 & 8,1 & 2,1 \\
\hline Schweden & 3,7 & 11 & 3,0 \\
\hline Deutschland & 3,6 & 11 & 3,1 \\
\hline Spanien & 3,5 & 4,9 & 1,4 \\
\hline Dänemark & 3,4 & 14,8 & 4,4 \\
\hline Israel & 3,4 & 4,5 & 1,3 \\
\hline Italien & 3,4 & 6,4 & 1,9 \\
\hline Frankreich & 3,3 & 8,2 & 2,5 \\
\hline Estland & 3,3 & 6,1 & 1,8 \\
\hline Australien & 3 & 10,2 & 3,4 \\
\hline Ungarn & 3 & 6,2 & 2,1 \\
\hline Niederlande & 2,9 & 8,4 & 2,9 \\
\hline Finnland & 2,7 & 9,6 & 3,6 \\
\hline Grossbritannien & 2,7 & 9,7 & 3,6 \\
\hline Luxemburg & 2,7 & 10,9 & 4,0 \\
\hline Neuseeland & 2,6 & 10,5 & 4,0 \\
\hline Slowenien & 2,4 & 8,1 & 3,4 \\
\hline USA & 2,4 & 10,8 & 4,5 \\
\hline Kanada & 2,4 & 9,4 & 3,9 \\
\hline Japan & 2,2 & 9,5 & 4,3 \\
\hline Polen & 2,2 & 5,2 & 2,4 \\
\hline Mexiko & 2 & 2,5 & 1,3 \\
\hline Südkorea & 1,9 & 4,5 & 2,4 \\
\hline Brasilien & 1,8 & 0,9 & 0,5 \\
\hline China & 1,4 & 1,4 & 1,0 \\
\hline Südafrika & 0,7 & 2,2 & 3,1 \\
\hline Indien & 0,7 & 0,9 & 1,3 \\
\hline Indonesien & 0,2 & 1,4 & 7,0 \\
\hline Tschechien & & 8,1 & \\
\hline Irland & & 12,7 & \\
\hline Durchschnitt & 2,8 & 7,7 & 2,8 \\
\hline
\end{tabular}

Bei internationalen Vergleichen der Zahl der Pflegefachleute ist zu berücksichtigen, dass Vollzeitäquivalente aussagekräftiger wären und dass teilweise noch immer unterschiedliche Definitionen angewendet werden. Verbesserungen der Pflegestatistiken sind aber im Gang, national und international [7]. Sie sind auch dringend nötig als Basis für Reformen und auch für Sparmassnahmen. Zudem ist der zahlenmässig grösste Beruf im Gesundheitssystem ein erheblicher Kostenblock.

\section{Grosse nationale Unterschiede: Beispiel Medizintechnik}

In der Gesundheitswesenforschung ist einer der wichtigsten Ansätze die Analyse von Unterschieden zwischen Ländern, Regionen, Städten und Krankenhäusern. Besonders häufig untersucht werden neben Bestandesgrössen (wie Ärzte- und Bettendichte) diagnostische Massnahmen, Operationen und Medikamentenkonsum. Auch wenn man sich auf kleinere, gut vergleichbare Regionen beschränkt, sind diese small area variations meistens viel grösser als angenommen und oft fast unbegreiflich (Beispiele bei www.dartmouthatlas.org).

Die Tabelle 4 zeigt Unterschiede in den 24 bis 32 erfassten OECD-Ländern. Danach variiert die Computertomographen-Dichte um das 23-fache und die Häufigkeit von drei Eingriffen um das 3- bis 71-fache. Die Schweiz hat die fünfthöchste CT-Dichte. In der Häufigkeit der drei Eingriffe auf Tabelle 4 ist die Schweiz im zweiten, dritten und siebenten Rang. Leider liefert die Schweiz der OECD keine Zahlen über MRI, im Gegensatz zu 30 anderen OECD-Ländern. Nach COCIR [8] hat unser Land die zweithöchste MRI-Dichte nach Japan und vor den USA. Dies ist mehr als doppelt so hoch wie der OECD-Durchschnitt von 12,2 Geräten pro Million Einwohner. Die Schweizer Ränge 2, 3, 5 und 7 (Spalte 2) illustrieren den hohen Versorgungsgrad in unserem Gesundheitswesen und sind auch einer der Gründe der hohen Ausgaben für unser Medizinsystem [9].

\section{Immer noch lange Aufenthaltsdauer im Spital - aber Kürzung naht dank DRG}

Krankenhaus-Kenndaten von 16 Ländern enthält die Tabelle 5. Auffällig ist in der Spalte 11 der zweite

Tabelle 4

Häufigkeit von vier Eingriffen, 2009. Erfasst: 24-32 Länder.

\begin{tabular}{|c|c|c|c|c|c|c|c|c|}
\hline & $\begin{array}{l}\text { Schweiz } \\
\text { Rang }\end{array}$ & $\begin{array}{l}\text { Schweiz } \\
\text { Zahl }\end{array}$ & $\begin{array}{l}\text { OECD } \\
\text { Durchschnitt }\end{array}$ & $\begin{array}{l}\text { OECD } \\
\text { Am häufigsten }\end{array}$ & $\begin{array}{l}\text { OECD } \\
\text { Am wenigsten häufig }\end{array}$ & $\begin{array}{l}\text { OECD } \\
\text { Maximum }\end{array}$ & $\begin{array}{l}\text { OECD } \\
\text { Minimum }\end{array}$ & $\begin{array}{l}\text { Verhältnis } \\
\text { Max./Min. }\end{array}$ \\
\hline Computertomographen * & 5 & 32,8 & 22,2 & Japan, Südkorea, Island & Mexiko, Ungarn, Israel & 97,3 & 4,3 & 23 \\
\hline Hüftgelenkersatz ** & 2 & 287 & 154 & $\begin{array}{l}\text { Deutschland, Schweiz, } \\
\text { Belgien }\end{array}$ & Mexiko, Südkorea, Chile & 296 & 8 & 37 \\
\hline Kniegelenkersatz ** & 3 & 200 & 118 & $\begin{array}{l}\text { Deutschland, USA, } \\
\text { Schweiz }\end{array}$ & Mexiko, Chile, Irland & 213 & 3 & 71 \\
\hline Kaiserschnitt *** & 7 & 32,4 & 26,3 & Brasilien, China, Türkei & $\begin{array}{l}\text { Niederlande, Finnland, } \\
\text { Island }\end{array}$ & 47,4 & 14,3 & 3 \\
\hline
\end{tabular}

* Geräte pro 1 Mio. Einwohner / ** pro 100000 Einwohner / *** pro 100 Lebendgeburten 
Tabelle 5

Krankenhaus-Kennzahlen, 2009 (teilweise 2008).

\begin{tabular}{|c|c|c|c|c|c|c|c|c|c|c|c|c|}
\hline \multirow[t]{2}{*}{ Land } & \multicolumn{2}{|c|}{$\begin{array}{l}\text { Spitäler pro } 1 \\
\text { Million E. }\end{array}$} & \multicolumn{2}{|c|}{$\begin{array}{l}\text { Betten } \\
\text { pro } 1000\end{array}$} & \multicolumn{2}{|c|}{$\begin{array}{l}\text { Personal- } \\
\text { dichte (3) }\end{array}$} & \multicolumn{2}{|c|}{$\begin{array}{l}\text { Akutbetten- } \\
\text { belegung }\end{array}$} & \multicolumn{2}{|c|}{$\begin{array}{l}\text { Aufenthaltsdauer } \\
\text { (alle Spitäler), }\end{array}$} & \multicolumn{2}{|c|}{$\begin{array}{l}\text { Krankenhausaus- } \\
\text { tritte je } 100000 \mathrm{E} .\end{array}$} \\
\hline & & Rang & Einw. & Rang & & Rang & in \% & Rang & Tage & Rang & gerundet & Rang \\
\hline Australien (2) & 61,3 & 1 & 3,8 & 7 & 13,8 & 7 & & & 6 & 7 & 16200 & 9 \\
\hline Dänemark & & & 3,5 & 9 & 18,5 & 1 & & & 4,8 & 12 & 17000 & 6 \\
\hline Deutschland & 40,5 & 5 & 8,2 & 1 & 11 & 10 & 76,2 & 9 & 9,7 & 2 & 23700 & 3 \\
\hline Finnland (2) & 58,2 & 2 & 6,2 & 4 & & & & & 12,5 & 1 & 18400 & 4 \\
\hline Frankreich (1) & 47,6 & 3 & 6,6 & 3 & 17,9 & 3 & 74,4 & 10 & 5,6 & 10 & 26400 & 2 \\
\hline Grossbritannien & & & 3,3 & 10 & & & 84,2 & 5 & 7,7 & 3 & 13800 & 10 \\
\hline Irland (2) & 39,8 & 6 & 4,9 & 6 & 11,4 & 9 & 89,2 & 3 & 6,1 & 6 & 13200 & 11 \\
\hline Italien & 21,4 & 8 & 3,7 & 8 & & & 79,5 & 6 & 6,7 & 5 & 13000 & 13 \\
\hline Kanada (2) & 21 & 9 & 3,3 & 10 & & & 93 & 1 & 7,7 & 3 & 8400 & 16 \\
\hline Niederlande & 11,1 & 13 & 4,7 & 7 & 12 & 8 & & & 5,8 & 8 & 11700 & 14 \\
\hline Norwegen (1) & 13,8 & 12 & 3,3 & 10 & 18,2 & 2 & 91,6 & 2 & 4,6 & 13 & 17700 & 5 \\
\hline Österreich (2) & 32 & 7 & 7,7 & 2 & 15,8 & 6 & 79 & 7 & 6,7 & 5 & 26600 & 1 \\
\hline Schweden (2) & & & 2,8 & 13 & & & & & 5,7 & 9 & 16600 & 8 \\
\hline Schweiz & 41,6 & 4 & 5,1 & 5 & 17,8 & 4 & 87,9 & 4 & 9,7 & 2 & 16800 & 7 \\
\hline Spanien & 16,8 & 11 & 3,2 & 11 & & & 77,6 & 8 & 6,9 & 4 & 10400 & 15 \\
\hline USA (1) & 19,1 & 10 & 3,1 & 12 & 16,9 & 5 & 65,5 & 11 & 4,9 & 11 & 13100 & 12 \\
\hline Durchschnitt & 32,6 & & 4,6 & & 15,3 & & 81,6 & & 6,9 & & 16400 & \\
\hline
\end{tabular}

(1) Austritt am Eintrittstag zählt als Krankenhausaustritt. (2) Austritt gesunder Neugeborener zählt nicht als Krankenhausaustritt. Würde Austritte um 3-6\% erhöhen. (3) Beschäftigte (Vollzeitäquivalente) in Krankenhäusern per 1000 Einwohner.

Rang der Schweiz (gemeinsam mit Deutschland) bezüglich Verweildauer in Krankenhäusern (alle Typen). Sie ist bei uns mit 9,7 Tagen 41\% länger als der Durchschnitt der hier aufgeführten Länder. Die kürzlich eingeführten Fallpauschalen werden wohl zusammen mit anderen Entwicklungen eine starke Angleichung der Aufenthaltsdauern an die anderen Länder bringen.

\section{0 statt 300 Krankenhäuser?}

Topografie, Reformschwäche und Extremföderalismus sind Hauptgründe, dass unsere Spitaldichte 28\% höher ist als im Vergleichsdurchschnitt (Spalte 2 der Tabelle 5). Die Personaldichte ist 16\% höher (Spalte 6). Eine Überschlagsrechnung zeigt, dass wir ein Spital auf $122 \mathrm{~km}^{2}$ haben, übertroffen nur von Deutschland mit $106 \mathrm{~km}^{2}$. Dort hat die Hälfte aller Krankenhäuser gemäss Michael M. Porter weniger als 200 Betten (Frankreich 54\%, Schweiz 72\% aller Akutspitäler). Das durchschnittliche Spitaleinzugsgebiet in den 13 erhobenen Ländern ist $2300 \mathrm{~km}^{2}$ (19-mal grösser als in der Schweiz). Bezieht man nur die europäischen Länder ein und damit nicht die drei Flächenstaaten Kanada, USA und Australien, ist der Durchschnitt immer noch $811 \mathrm{~km}^{2}$ pro Spital. Nähme die Schweiz diese Fläche als Norm - Gedankenspiele sind ja nicht verboten und Spitalplanung ist hochaktuell -, müssten wir über 280 unserer 336 Krankenhäuser schliessen. Die alte Forderung «50 Spitäler genügen!» ist also theoretisch nicht so abwegig, wie sie empfunden wurde, als sie vor rund zehn Jahren erstmals auftauchte [10]. In der Praxis allerdings wäre schon die Schliessung von 50 Betrie- ben eine Sensation. Die Tendenz ist aber unausweichlich und wird unser Gesundheitssystem massiv umstrukturieren.

\section{Literatur}

1 Health at a glance 2011. OECD Indicators. OECD. Paris; 2011.

2 http://stats.oecd.org/Index. aspx?DatasetCode=HEALTH_STAT. Siehe auch: www.oecd-ilibrary.org

3 Statistik der obligatorischen Krankenversicherung. 2009. BAG; 2011.

4 Straub M, Hartwig J. Herbstprognose der schweizerischen Gesundheitsausgaben 2010-2013. KOF/ETH 2011.

5 s. dazu: Frey M et al. Direkte Gesundheitsausgaben der privaten Haushalte. Out-of-Pocket-Zahlungen und Kostenbeteiligungen in der Schweiz und im internationalen Vergleich. Schweizerisches Gesundheitsobservatorium (Obsan). Neuenburg; 2011.

6 Ausführlicher dazu: Kocher G. Internationale Vergleiche von Kosten und Qualität. Schweiz Ärztezeitung. 2010;91(42):1663-7.

7 Rossel R. Personnel soignant en Suisse. Sources et méthodes de l'estimation des ressources humaines engagées dans le système de santé selon la qualification. Office fédéral de la statistique. 10 février 2010.

8 Comité européen de coordination des industries radiologiques, électromédicales et d'informatique de santé. Brüssel.

9 s. dazu: Kocher G. Die Gründe der «Kostenexplosion». Schweiz Ärztezeitung. 2011;92(38):1466-9.

10 de Wolff F. (2002), später u. a. Manfred Manser, Willy Oggier, Thomas Zeltner, Pascal Couchepin. 